\title{
Marcadores sorológicos da hepatite B em usuários de um Centro de Testagem para o HIV
}

\author{
Serological markers of hepatitis B in people attending a \\ HIV Counseling and Testing Center
}

\begin{abstract}
Maria Rita de Cassia Costa Monteiro', Afonso Dinis Costa Passos², José Fernando de Castro Figueiredo ${ }^{3}$, Ana Maria Coimbra Gaspar ${ }^{4}$ e Clara Fumiko Tachibana Yoshida ${ }^{4}$
\end{abstract}

\begin{abstract}
Resumo Esta investigação objetivou estudar a prevalência de marcadores sorológicos de infecção pelo vírus da hepatite $B$ e analisar possíveis fatores de risco em 404 usuários submetidos à sorologia anti-HIV no Centro de Testagem e Aconselhamento de Ribeirão Preto, SP, Brasil. A prevalência global dos marcadores para o vírus da hepatite $B$ foi de $14,6 \%$, idêntica à encontrada para o anti-HBc, com valores de $1 \%$ para 0 HBsAg e anti-HBc lgM. Após ajuste por regressão logística, os marcadores de infecção do vírus $B$ mostraram associação com as variáveis: idade, local de residência, uso de drogas endovenosas e positividade para o HIV. A prevalência de infecção pelo vírus da imunodeficiência humana foi de $6,9 \%$. Marcadores do vírus $B$ foram detectados em 55,6\% dos usuários de drogas endovenosas e em $42,9 \%$ dos positivos ao vírus da imunodeficiência humana, confirmando altos índices de infecção nestes grupos específicos.
\end{abstract}

Palavras-chaves: Hepatite B. Epidemiologia. Fatores de risco. Coinfecção.

Abstract The objective of this investigation was to study the prevalence of serological markers of hepatitis $B$ and possible risk factors for this disease in a sample of 404 people who attended a Testing and Couseling Center for HIV in the city of Ribeirão Preto, São Paulo State, Brazil. The overall prevalence of serologic hepatitis B markers was $14.6 \%$, equal to that obtained for anti-HBc. HBsAg and anti-HBc IgM showed prevalences of $1 \%$. After adjustment using logistic regression, hepatitis $B$ markers showed association with the following variables: age, place of residence, use of injectable drugs and positivity to anti-HIV. The overall prevalence of human immunodeficiency virus infection was $6.9 \%$. Hepatitis B markers were detected in $55.6 \%$ among intravenous drug users and in $42.9 \%$ among those who tested positive for HIV, confirming literature findings which indicates high levels of infection in these specific population groups.

Key-words: Hepatitis B. Epidemiology. Risk factors. Coinfection.

Muito embora largamente difundida no mundo, a hepatite $\mathrm{B}$ mostra nítidas diferenças geográficas que se manifestam tanto na incidência da doença aguda quanto na prevalência da infecção e na freqüência da doença crônica ${ }^{12481019}$. Essa diversidade no padrão epidemiológico pode ser atribuída a fatores biológicos, ambientais e mesmo sociais. Todavia, este padrão vem sofrendo evidentes transformações nas duas últimas décadas ${ }^{1}$. O surgimento de uma vacina nos anos 80 , e as mudanças de comportamento de alguns grupos populacionais podem, em parte, justificar essas mudanças.
Um estudo realizado na população americana nos anos de 1982 a 1988, demonstra um crescimento significativo na proporção de casos de hepatite B entre usuários de drogas endovenosas e heterossexuais, com redução no grupo de homossexuais masculinos, trabalhadores na área da saúde em contato freqüente com sangue e naqueles com história de transfusão de sangue ${ }^{1}$.

O conhecimento da distribuição do vírus da hepatite B (HBV) no Brasil ainda é superficial, a despeito da gravidade da doença, especialmente em suas formas crônicas. Estudos de prevalência se fazem necessários,

\footnotetext{
1. Departamento de Patologia Tropical do Curso de Medicina, Centro de Ciências da Saúde, Universidade Federal do Pará, Belém, PA, Brasil. 2. Departamento de Medicina Social da Faculdade de Medicina de Ribeirão Preto, Universidade de São Paulo, SP, Brasil. 3. Divisão de Moléstias Infecciosas e Tropicais. Departamento de Clínica Médica da Faculdade de Medicina de Ribeirão Preto, Universidade de São Paulo, SP, Brasil. 4. Centro de Referência Nacional para Hepatites Virais, Instituto Oswaldo Cruz. Fiocruz. Rio de Janeiro, RJ, Brasil.

Endereço para correspondência: Maria Rita de Cassia Costa Monteiro. Rua São Miguel 560 apto. 901, Batista Campos, $66033-010$ Belém, Pará, Brasil Fax: $5591252-4136$

e-mail monteiro@amazon.com.br

Recebido para publicação em 2/6/99.
} 
de modo especial em populações com maior risco, não só pelo conhecimento ainda pouco abrangente que se tem sobre a situação do HBV nesses grupos, mas também pelo aumento crescente do número de indivíduos em situações que favorecem o contágio, como os usuários de drogas endovenosas, por exemplo. Outro importante aspecto ainda pouco conhecido entre nós é a freqüência da co-infecção do HBV com o vírus da imunodeficiência humana (HIV), tendo em vista que a possibilidade de associação destas viroses em um mesmo indivíduo está facilitada, em parte, pela semelhança nas formas de transmissão, permitindo que sejam comuns entre eles um grande número de situações consideradas de risco.

A presente investigação foi realizada visando caracterizar alguns aspectos do padrão epidemiológico do HBV em indivíduos com risco de infecção pelo HIV. Seus objetivos específicos foram estudar a prevalência dos marcadores de infecção pelo vírus $\mathrm{B}$, bem como alguns fatores de risco a ela associados, em indivíduos submetidos à sorologia anti-HIV no Centro de Testagem e Aconselhamento (CTA) da cidade de Ribeirão Preto, SP, local para onde acorre um grande número de indivíduos que se consideram sob risco de infecção pelo HIV.

\section{MATERIAL E MÉTODOS}

Definiu-se como população de referência todos os indivíduos atendidos no CTA e submetidos à sorologia anti-HIV. Uma amostra dessa população, atendida no período entre maio e agosto de 1998, foi definida como população de estudo. Assim, foram incluídos todos os indivíduos submetidos à coleta de sangue para realização de sorologia anti-HIV no CTA, no período da pesquisa, e que consentiram por escrito na sua participação, após esclarecimento verbal sobre os objetivos e metodologia do trabalho em foco. Neste Centro, são atendidos indivíduos maiores de 13 anos de idade que o procuram espontaneamente para realização de sorologia anti-HIV. O tamanho da amostra foi calculado com base na fórmula utilizada para determinação do $n$ em levantamentos: $n=Z^{2} P Q / d^{2}$. Assumindo um alfa de 0,05 , uma precisão de $5 \%$ e uma prevalência estimada de marcadores de infecção pelo HBV da ordem de $50 \%$, o tamanho amostral mínimo determinado foi de 384 indivíduos. Ao final, participaram do estudo 404 indivíduos.

Tanto o convite à participação quanto o repasse das informações sobre os objetivos e procedimentos adotados na pesquisa foram realizados por um dos autores, durante a reunião diária que se faz com os usuários presentes no CTA. Para obtenção das informações relativas às características individuais e fatores de risco para hepatite $\mathrm{B}$ foi aplicado à cada participante um questionário padrão. Simultaneamente, no momento da punção venosa para coleta do sangue destinado à sorologia anti-HIV, fez-se uma coleta adicional de $8 \mathrm{ml}$ de sangue para a pesquisa dos marcadores do HBV. Os procedimentos para realização da sorologia anti-HIV seguiram a orientação fornecida pela Secretaria de Vigilância Sanitária do Ministério da Saúde do Brasil e que obedecem uma seqüência em três etapas $^{16}$. Na etapa I, os soros foram examinados em duplicata, através de ensaios imunoenzimáticos de segunda e terceira geração, com os reativos: Genscreen ${ }^{\circledR}$ HIV 1/2 produzido pela Sanofi Diagnostics Pasteur SA; Genetic Systems $^{\mathrm{TM}}$ LAV EIA, fabricado pela Genetic Systems Corporation e Abbott HIV-1/HIV-2 $3^{\text {rd }}$ generation plus EIA, produzido pela Abbott Diagnostic Division. Para leitura dos resultados com os reativos da Abbott utilizou-se espectrofotômetro Quantum II; para os outros foi utilizado leitor de microplaca LP 400 (Sanofi Pasteur). Definiu-se o cutoff (ponto de corte) de acordo com a orientação do fabricante. No teste de Imunoflorescência Indireta (etapa II) foram usados os reagentes fornecidos pelo Instituto de Tecnologia em Imunobiológicos - Bio Manguinhos, da Fiocruz, seguindo metodologia adotada pelo mesmo ${ }^{17}$. Na leitura desta reação foi utilizado um microscópio para fluorescência Nikon, tipo Labophot-2. O teste Western Blot (etapa III) foi executado com os seguintes reativos comerciais: HIV-1 blot 1.3, produzido pela Genelabs Diagnostics; Cambridge biotech HIV-1 da Cambridge Biotech Corporation, e o HIV1 Western Blot da Organon Teknika Corporation. A determinação da positividade foi feita com base numa tabela de pontuação e de critérios fornecida pelo fabricante.

Na pesquisa de infecção pelo HBV foram investigados os seguintes marcadores sorológicos: $\mathrm{HBsAg}$, anti-HBc $\mathrm{IgM}$, anti-HBc e, nas amostras reagentes ao anti-HBc, realizou-se a pesquisa do anti-HBs. Para detecção destes marcadores, todas as amostras de soro foram submetidas a exames imunoenzimáticos executados no Centro de Referência Nacional para Hepatites Virais, do Departamento de Virologia do Instituto Oswaldo Cruz-Fiocruz. Todos estes exames foram realizados com reagentes da linha Hepanostika ${ }^{\circledR}$ (HBsAg Uni-Form II, anti-HBs New, antiHBc IgM e anti-HBc Uni-Form), fabricados pela Organon Teknika. Para leitura dos resultados utilizou-se o leitor de ELISA da Organon Teknika (Microwell System - Reader 230). Os procedimentos técnicos referentes aos testes imunológicos foram realizados de acordo com as especificações dos fabricantes dos reagentes e dos aparelhos empregados. Foram considerados positivos para o HBV todos os indivíduos com um ou mais resultados reagentes aos marcadores pesquisados.

O projeto desta pesquisa foi aprovado pela Comissão de Ética da Secretaria Municipal de Saúde de Ribeirão Preto e pela Comissão de Ética em Pesquisa do Hospital das Clínicas da Faculdade de Medicina de Ribeirão Preto, da Universidade de São Paulo.

A abordagem estatística inicial consistiu de uma análise univariada, mediante a utilização dos testes quiquadrado, qui-quadrado para tendência e teste exato de Fisher, buscando-se associação entre as possíveis variáveis independentes e a positividade sorológica para 
o HBV. As variáveis que demonstraram um valor de $\mathrm{p}<$ 0,15 foram incluídas em um modelo de regressão logística não condicional, tendo sido previamente testadas para a ocorrência de interação. Em todas as situações, o limite de significância estatística adotado foi igual a 0,05 .

\section{RESULTADOS}

A média de idade dos indivíduos pesquisados foi de 29,7 anos, com desvio padrão de 11,4 e mediana de 27 anos.

Na Tabela 1 verifica-se a distribuição da prevalência global dos marcadores sorológicos de infecção pelo HBV de acordo com o sexo e faixa etária. Foram positivos ao anti-HBc 59 indivíduos, determinando uma prevalência para este marcador de 14,6\% (IC 95\%: 11,2-18), idêntica à prevalência global do HBV, dado que todos os indivíduos reagentes aos outros marcadores foram positivos a ele. A positividade entre os sexos mostrou valores iguais a $16,5 \%$ e $12,7 \%$, respectivamente para os homens e as mulheres $(p=0,353)$. Os valores da prevalência de infecção pelo HBV mostraram uma tendência ascendente até a faixa de 50 a 59 anos, reduzindo-se entre os indivíduos mais idosos $\left(\chi^{2}\right.$ para tendência $\left.=10,957 ; p=0,000\right)$. Embora

Tabela 1 - Distribuição da prevalência global dos marcadores sorológicos do HBV na amostra investigada, de acordo com o sexo e faixa etária.

\begin{tabular}{|c|c|c|c|c|c|c|c|c|c|}
\hline \multirow{3}{*}{ Idade } & \multicolumn{9}{|c|}{ Sexo } \\
\hline & \multicolumn{3}{|c|}{ masculino } & \multicolumn{3}{|c|}{ feminino } & \multicolumn{3}{|c|}{ total $^{*}$} \\
\hline & $\mathrm{n}^{\circ}$ & + & $\%$ & $\mathrm{n}^{\circ}$ & + & $\%$ & $\mathrm{n}^{\circ}$ & + & $\%$ \\
\hline $14|-| 19$ & 37 & 1 & 2,7 & 33 & 1 & 3,8 & 70 & 2 & 2,9 \\
\hline $20|-| 29$ & 91 & 15 & 16,5 & 84 & 9 & 10,7 & 175 & 24 & 13,7 \\
\hline $30|-| 39$ & 46 & 12 & 26,1 & 50 & 7 & 14,0 & 96 & 19 & 19,8 \\
\hline $40|-| 49$ & 9 & 2 & 22,2 & 24 & 3 & 12,5 & 33 & 5 & 15,2 \\
\hline $50|-| 59$ & 6 & 1 & 16,7 & 12 & 6 & 50,0 & 18 & 7 & 38,9 \\
\hline $60|-| 79$ & 11 & 2 & 18,2 & 1 & 0 & 0,0 & 12 & 2 & 16,7 \\
\hline Total & 200 & 33 & 16,5 & 204 & 26 & 12,7 & 404 & 59 & 14,6 \\
\hline
\end{tabular}

ํo = número de indivíduos pesquisados. + = indivíduos com um ou mais resultados positivos. * $=$ prevalência global.

não mostrado em tabela, observou-se positividade ao HBsAg em 4 pacientes, o mesmo ocorrendo para o anti$\mathrm{HBc}$ lgM, o que resultou numa prevalência de $1 \%$ para ambos. A pesquisa do anti-HBs se fez apenas nos 59 indivíduos positivos ao anti-HBc, sendo observada sua positividade em 33 pacientes (55,9\%).

No conjunto investigado, 31,2\% (126) informaram ter feito uso de qualquer tipo de droga ilícita (injetável ou não).
Conforme mostrado na Tabela 2, no grupo que relatou uso atual ou pregresso de droga ilícita endovenosa (9), a prevalência de marcadores de hepatite B foi de $55,6 \%$, contra $13,7 \%$ entre os não usuários ( $p=0,004)$.

A Tabela 3 evidencia os 28 indivíduos positivos para o HIV presentes na amostra, o que resultou em uma prevalência para este vírus de 6,9\% (IC: 4,4 - 9,3). A taxa de infecção pelo HBV foi de $42,9 \%$ nos soropositivos e de

Tabela 2 - Distribuição da prevalência global de marcadores de infecção pelo HBV de acordo com uso passado ou atual de drogas ilícitas endovenosas.

\begin{tabular}{|c|c|c|c|c|c|c|}
\hline \multirow{3}{*}{$\begin{array}{l}\text { Uso de droga } \\
\text { ilícita endovenosa }\end{array}$} & \multicolumn{6}{|c|}{ HBV } \\
\hline & \multicolumn{2}{|c|}{ positivo } & \multicolumn{2}{|c|}{ negativo } & \multicolumn{2}{|c|}{ total } \\
\hline & $\mathrm{n}^{\circ}$ & $\%$ & $\mathrm{n}-0$ & $\%$ & no & $\%$ \\
\hline Sim & 5 & 55,6 & 4 & 44,4 & 9 & 100,0 \\
\hline Não & 54 & 13,7 & 341 & 86,3 & 395 & 100,0 \\
\hline Total & 59 & 14,6 & 345 & 85,4 & 404 & 100,0 \\
\hline
\end{tabular}

Tabela 3 - Distribuição da prevalência global dos marcadores de infecção pelo HBV de acordo com a sorologia anti-HIV.

\begin{tabular}{|c|c|c|c|c|c|c|}
\hline \multirow{3}{*}{$\begin{array}{l}\text { Sorologia } \\
\text { anti-HIV }\end{array}$} & \multicolumn{6}{|c|}{ HBV } \\
\hline & \multicolumn{2}{|c|}{ positivo } & \multicolumn{2}{|c|}{ negativo } & \multicolumn{2}{|c|}{ total } \\
\hline & $\mathrm{n}^{0}$ & $\%$ & $\mathrm{n}^{0}$ & $\%$ & $\mathrm{n}^{0}$ & $\%$ \\
\hline Positivo & 12 & 42,9 & 16 & 57,1 & 28 & 100,0 \\
\hline Negativo & 47 & 12,5 & 329 & 87,5 & 376 & 100,0 \\
\hline Total & 59 & 14,6 & 345 & 85,4 & 404 & 100,0 \\
\hline
\end{tabular}


$12,5 \%$ nos soronegativos ao HIV ( $p=0,000)$. A co-positividade entre o HBV e o HIV foi da ordem de $3 \%$ (12/404).

$\mathrm{Na}$ Figura 1 observam-se que as prevalências do HBV superam as do HIV em todas as faixas etárias.
Além disso, verifica-se uma distribuição diferenciada das duas infecções no que diz respeito a idade, com predomínio de indivíduos mais jovens entre os positivos ao HIV e de mais idosos entre os infectados pelo HBV.

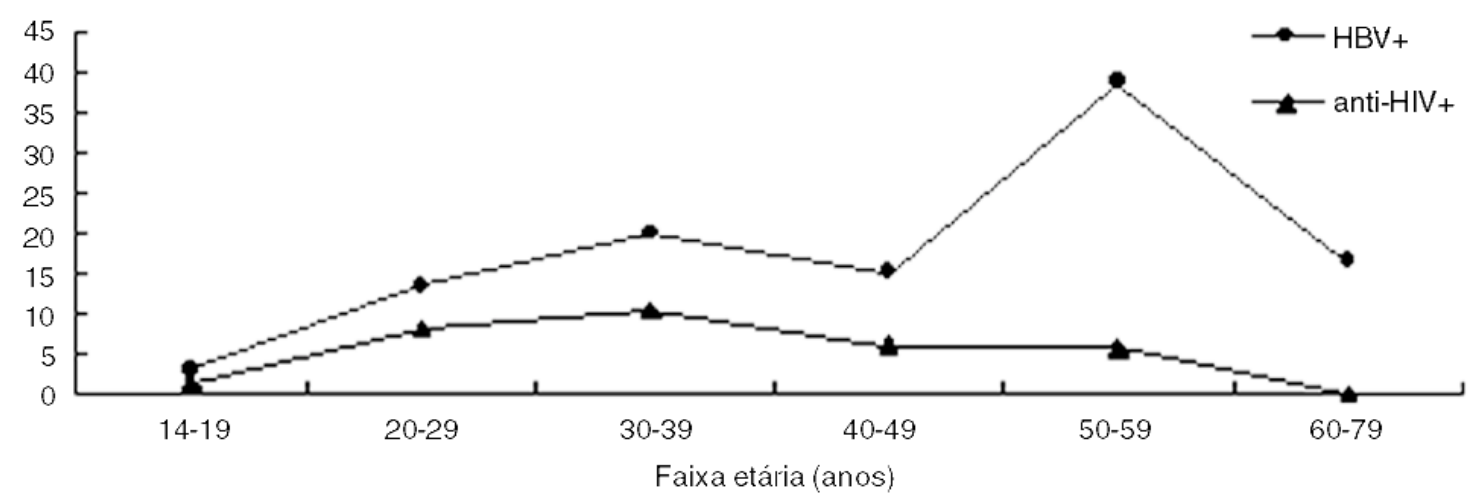

Figura 1 -Distribuição dos reagentes a o HBV e à sorologia anti-HIV por faixa etária.

Embora não representados em tabelas ou figuras, diversos outros achados do trabalho merecem menção.

Assim, na investigação da escolaridade observou-se, através da análise univariada, positividade aos marcadores do HBV em $22,8 \%$ dos 79 indivíduos com menos de 4 anos de escolaridade e em $12,6 \%$ das 325 pessoas com 4 ou mais anos $(p=0,034)$. Entre os 230 que referiam habitar domicilio próprio $14,8 \%$ foram positivos para algum marcador do HBV, enquanto que nos 174 sem domicílio próprio esta taxa foi de $14,4 \%(p=0,979)$. A análise da situação ocupacional revelou uma positividade de $13,5 \%$ entre os 185 indivíduos desempregados, donas de casa, estudantes e em outras situações não classificadas; nos empregados ou trabalhando por conta própria (211) a prevalência foi de $14,7 \%$, enquanto nos 8 indivíduos aposentados o valor atingiu $37,5 \%(p=0,170)$.

$\mathrm{Na}$ avaliação da renda familiar mensal encontrou-se $15,9 \%$ de positividade para algum marcador do HBV entre os 170 indivíduos com renda menor que cinco salários mínimos e de 13,7\% nos 234 indivíduos com renda igual ou superior a cinco salários mínimos $(p=0,633)$.

A prevalência de positividade ao HBV foi de $28,6 \%$ entre os 21 indivíduos com antecedente de icterícia presentes na amostra e de $14,2 \%$ entre os 383 que não referiam este antecedente $(p=0,102)$. No que diz respeito à história prévia de hepatite, as prevalências de HBV foram de $26,1 \%$ nos 23 participantes com tal antecedente e de $14 \%$ entre os 381 que não o apresentavam ( $p=0,125)$.

Foram positivos para o HBV 25\% dos 104 nascidos em área rural e $11 \%$ dos 300 nascidos em área urbana $(p=0,000)$. Nos residentes em Ribeirão Preto (312), a taxa de infecção pelo HBV foi $16,3 \%$, enquanto que nos 92 não residentes este valor alcançou $8,7 \%(p=0,097)$.

No grupo investigado, 54 indivíduos referiram ocupação, atual ou anterior, envolvendo contato com sangue, e nestes a positividade para o HBV foi 18,5\%. Entre os que negaram essa atividade (350), a freqüência de positivos foi de $14 \%(p=0,504)$. Em relação ao antecedente de transfusão de sangue e/ou hemoderivados, os valores de prevalência foram de 19,4\% naqueles 36 indivíduos com tal história e de $14,1 \%$ nos 368 que a negavam ou a ignoravam $(p=0,538)$.

Nos participantes que relataram comportamento heterossexual (356) a prevalência dos marcadores do HBV foi de $13,8 \%$, com valores respectivamente iguais a $18,2 \%$ nos 11 de comportamento homossexual e 22,2\% nos 36 bissexuais. Um indivíduo não informou sua preferência sexual, e neste a pesquisa desses marcadores foi negativa. O agrupamento desta variável em duas categorias (heterossexual/sem informação da preferência sexual e homossexual/bissexual), não produziu diferença estatística significante $(p=0,246)$.

Entre aqueles já com experiência sexual (403), somente $50(12,4 \%)$ referiram usar sempre preservativo nas relações sexuais, e entre eles a prevalência dos marcadores para o HBV foi $16 \%$. Tal prevalência foi de $14,4 \%$ no grupo de 353 pessoas que informou usar preservativo esporadicamente ou nunca usá-lo ( $p=0,938)$.

A pesquisa de antecedente de doença sexualmente transmissível, no grupo com experiência sexual prévia, revelou uma positividade de marcadores do HBV de $23,3 \%$ entre os 90 participantes com história de tais doenças. Entre os 313 indivíduos que não as relatavam, o valor foi de $12,1 \%$, $(p=0,013)$.

Tomando-se todas as variáveis que mostraram um valor de $p<0,15$ na análise univariada (idade, uso de droga ilícita endovenosa, positividade anti-HIV, escolaridade, antecedente de icterícia e de hepatite, área urbana ou rural de nascimento, local de residência e antecedente 
de doença sexualmente transmissível) e, submetendoas a um modelo de regressão logística não condicional, observou-se existir associação significante entre a reatividade aos marcadores do $\mathrm{HBV}$ e as variáveis: idade, local de residência, uso de droga ilícita endovenosa e positividade ao HIV (Tabela 4).

Tabela 4 - Variáveis com valores significantes na análise final por regressão logística.

\begin{tabular}{lccc}
\hline Variável & Odds Ratio & IC 95\% & P \\
\hline Idade & 1,04 & $1,01-1,06$ & 0,000 \\
Local de residência & 2,41 & $1,03-5,61$ & 0,041 \\
Uso de droga endovenosa & 5,00 & $1,07-23,18$ & 0,040 \\
Positividade anti-HIV & 4,25 & $1,73-10,48$ & 0,002 \\
\hline
\end{tabular}

\section{DISCUSSÃO}

No presente estudo, embora examinando um grupo considerado com maior risco de infecção pelo HBV, a prevalência encontrada dos diversos marcadores sorológicos aproxima-se da observada em populações fora dos grupos com maior prevalência deste vírus ${ }^{14}$. Uma justificativa plausível para este fato, dado que a amostra foi retirada de uma população que procura um Serviço com a finalidade de conhecer sua situação sorológica para o HIV, freqüentemente por se achar sob risco, é que esteja ocorrendo, neste grupo, um entendimento maior de que a aids não está restrita exclusivamente a pessoas com determinados comportamentos. Conseqüentemente, indivíduos com potencial de risco menor podem estar procurando o Serviço, ocasionando uma redução da prevalência dos marcadores pesquisados. Um outro fator a ser considerado na análise destes resultados é a grande freqüência de jovens na população de estudo, com cerca de $85 \%$ situando-se entre 14 e 39 anos. Em regiões de baixa endemicidade para o vírus da hepatite $\mathrm{B}$, como é o caso da cidade de Ribeirão Preto, há uma tendência de crescimento da taxa de infecção do HBV que se faz proporcional à idade, fato este confirmado nos dados do presente estudo. Assim, a alta proporção de indivíduos jovens na amostra estudada pode estar contribuindo para uma prevalência aparentemente reduzida dos marcadores de hepatite $B$.

A utilização de drogas ilícitas constitui um problema crescente, tanto em regiões desenvolvidas como naquelas em desenvolvimento, estimando-se atualmente a existência de cerca de cinco milhões de usuários de drogas injetáveis no mundo ${ }^{11}$. A forte associação verificada nesta pesquisa entre o uso de droga ilícita endovenosa e a positividade para hepatite $B$ coincide com evidências derivadas de outras investigações ${ }^{1101321}$.

Cerca de 2/3 da população no presente estudo referiu uso de algum tipo de droga ilícita. A propósito, a cidade de Ribeirão Preto tem sido considerada um grande pólo de consumo de drogas no estado de São Paulo. Paralelamente, é um dos municípios com maior incidência de aids no Brasil, com os maiores índices se fazendo presentes nos usuários de drogas injetáveis ${ }^{15} 18$. A semelhança nas vias de transmissão do HBV e HIV pode justificar, de algum modo, o encontro de um risco maior de infeção pelo vírus da hepatite B entre os residentes em Ribeirão Preto, quando comparado aos que residem em outros locais.

Em regiões subdesenvolvidas, com baixa renda per capita, como a África e o Sudeste Asiático, a prevalência de marcadores de infecção pelo HBV entre a população adulta de algumas áreas chega a 100\%12. Embora considerando as dificuldades na obtenção e análise das informações referentes à situação sócioeconômica, procurou-se investigar no presente estudo dados relativos à escolaridade, posse de domicílio, situação ocupacional e renda familiar mensal, com a finalidade de observar o comportamento dos marcadores sorológicos do HBV frente a essas variáveis. Apenas a escolaridade mostrou diferenças significantes na análise univariada. Contudo, o controle dos fatores de confusão pela análise por regressão logística não confirmou este achado, evidenciando a não associação entre a condição econômica e a presença de hepatite B na amostra estudada.

A infecção aguda pelo HBV é silenciosa em mais da metade dos $\operatorname{casos}^{24}$, o que favorece a existência de uma grande parcela de indivíduos com marcadores positivos sem história prévia de doença aguda ${ }^{22}$. Nesta casuística, a freqüência de infecção entre os que referiram antecedente de icterícia correspondeu ao dobro da observada entre aqueles sem este antecedente, porém esta diferença não foi significante na análise final. O mesmo ocorreu na investigação do antecedente de hepatite. É provável, especialmente por tratar-se de um grupo com grande percentual de jovens, que muitos dos casos com antecedente de icterícia e/ou hepatite possam ter na sua etiologia o vírus da hepatite $A$, clinicamente indistinguível da doença causada pelo vírus $\mathrm{B}$.

Indivíduos com exposição ocupacional ao sangue, especialmente os trabalhadores na área da saúde, constituem um grupo de risco para o HBV, risco esse que aumenta quando atuam em áreas onde o contato com sangue é mais freqüente e prolongado ${ }^{36}$. Outras formas de contato casual nesses ambientes trazem riscos menores de infecção ${ }^{22}$, sendo esta, possivelmente, a forma de contato mais provável entre os integrantes 
desta pesquisa que referiram exposição ocupacional ao sangue, e que pode justificar a ausência de diferenças estatisticamente significantes na análise desta variável.

O não encontro, no presente estudo, de uma associação entre história de transfusão de sangue e positividade para o HBV, possivelmente traduza o controle adequado dos centros de hemoterapia no sentido de evitar a transmissão do HBV através dos produtos utilizados nas transfusões.

Os homossexuais constituem um grupo com risco maior de infecção pelo HBV. Em São Paulo, observou-se nesta população uma prevalência global para o HBV de 31,9\%, com 2,8\% para o $\mathrm{HBsAg}^{7}$. Em anos mais recentes, mudanças de comportamento têm sido observadas entre os homossexuais, e um dos itens citado como reflexo dessas transformações é o indício de estabilidade e redução dos casos de aids neste grupo. É de se esperar, portanto, que outras doenças freqüentes nesta população, como a hepatite $B$, também apresentem tendência de redução ${ }^{1}$, o que vem de encontro aos resultados deste trabalho, pois, embora se tenha encontrado prevalência mais baixa de infecção pelo HBV no grupo de comportamento heterossexual quando comparada a dos homossexuais, esta diferença não foi significante estatisticamente.
A despeito das inúmeras campanhas alertando sobre os riscos presentes nas relações sexuais desprotegidas, este hábito ainda não se consolidou no grupo investigado, dado que somente $12,4 \%$ dos participantes afirmaram sempre usar preservativo nas relações sexuais. A não observação de riscos diferenciados entre os que usam este recurso e os que não o fazem possivelmente denote a importância de outras vias de transmissão do HBV na população investigada.

A forte associação entre o HBV e o HIV, confirmada em inúmeras referências de literatura ${ }^{52023}$, provavelmente deriva-se da semelhança nas formas de transmissão e, conseqüentemente, dos fatores de risco. Esta associação deve ser lembrada quando das campanhas preventivas para o HIV, o que certamente ajudaria a reduzir o amplo desconhecimento de aspectos epidemiológicos fundamentais da hepatite B por parte da população. Apesar disso, é importante notar que nesta investigação a prevalência do HIV foi bem mais elevada $(6,9 \%)$ do que na população geral, a despeito do mesmo não ocorrer em relação ao HBV. Isto aponta para a existência de situações peculiares a cada uma dessas infecções, o que justifica a necessidade de mais estudos destinados a esclarecer outros aspectos da história natural de ambas as infecções.

\section{REFERÊNCIAS BIBLIOGRÁFICAS}

1. Alter MJ, Hadler SC, Margolis HS, Alexander WJ, Hu PY, Judson FN, Mares A, Miller JR, Moyer LA. The changing epidemiology of hepatitis $B$ in the United States. Need for alternative vaccination strategies. The Journal of the American Medical Association 263:1218-1222, 1990

2. Bensabath G, Soares MCP. A hepatite B e delta em Boca do Acre, Alto Purus. Moderna Hepatologia 14:36-40, 1989

3. Boccato RSBS. Avaliação da resposta imunológica à vacina contra a hepatite B aplicada pelas vias intradérmica ou intramuscular em profissionais da saúde de hospital universitário: seguimento de cinco anos. Tese de Doutorado, Universidade Estadual de Campinas, Campinas, SP, 1996.

4. Castro EJ, Rosa Filho SM. Hepatite B num hospital militar (prevalência e profilaxia): experiência do Hospital Central do Exército. Moderna Hepatologia 14:19-21, 1989.

5. Duarte G, Gir E, Machado AA, Figueiredo JFC, Martinez R. Correlación entre sífilis, hepatitis $\mathrm{B}$ y la infección por el virus de inmunodeficiencia adquirida tipo $1(\mathrm{VIH}-1)$. Revista IberoLatinoamericana de Enfermedades de Transmissión Sexual 10:1117, 1996.

6. Ferraz MLG, Silva AEB. Hepatitis B: a risk to the health care professional. Revista do Hospital São Paulo 1:140-144, 1989.

7. Figueiredo GM, Veras MA, Luna EJ. Prevalence and incidence of hepatitis $B$ and $C$ among men who have sex with men (MSM) in São Paulo, Brazil: the Bela Vista cohort study. International Conference AIDS 11: 457, 1996.

8. Galizzi FJ, Andrade MD, El Azzi SP, Torres RA, Barbosa WS, Simonetti JP, Simonetti SRR, Faria O de, Melo L, Nogueira AMFF, Bambirra EA. Hepatite em Belo Horizonte. Moderna Hepatologia 14:22-24, 1989.
9. Gonçales Jr FL, Pavan MHP, Aoki FH, Lazarini MSK, Gonçales NSL. Marcadores sorológicos para hepatite $B$ e hepatite $C$ em pacientes infectados pelo HIV-1. In: IX Congresso Brasileiro de Infectologia, Recife p.163, 1996.

10. Lurie P, Fernandes ME, Hughes V, Arevalo EI, Hudes ES, Reingold A, Hearst N. Socioeconomic status and risk of HIV-1, syphilis and hepatitis B infection among sex workers in São Paulo State, Brasil. AIDS 9(Suppl. I):S31-S37, 1995.

11. Martínez-Morales JM. $O$ avanço do Brasil nas questões relativas às drogas. Encarte do Boletim Epidemiológico AIDS 11:4-5, 1998.

12. Mendes TF. Carcinoma hepatocelular: Um tumor evitável? Editorial. Moderna Hepatologia 16:1, 1991.

13. Mendes TF, Pittella AM, Herbert BA. Hepatite B no Rio de Janeiro. Moderna Hepatologia 14:14-18, 1989.

14. Miranda LGV, Passos ADC, Figueiredo JFC, Gaspar AMC, loshida CFT. Marcadores sorológicos de hepatite $B$ em indivíduos submetidos a exames de sangue em Unidades de Saúde. Revista de Saúde Pública 34:286-291, 2000.

15. Ministério da Saúde. Coordenação Nacional de Doenças Sexualmente Transmissíveis e AIDS. Boletim epidemiológico AIDS, ano XI, n. 02, 1998.

16. Ministério da Saúde. Dispõe sobre a padronização dos procedimentos sequenciados para detecção de anticorpos antiHIV em indivíduos com idade acima de 2 anos. Diário Oficial da União. Brasília, Portaria n.488 de 17 de junho de 1998, 1998.

17. Ministério da Saúde. Instituto de Tecnologia em ImunobiológicosBio Manguinhos. Teste de Imunoflorescência Indireta (IFI) para diagnóstico da infecção pelo HIV-1. Fiocruz, Rio de Janeiro, fevereiro, 1998. 
18. Ortuza EM. Estudo epidemiológico sobre a síndrome de imunodeficiência adquirida (AIDS) no município de Ribeirão Preto, SP, Brasil. Dissertação de Mestrado, Universidade de São Paulo, Ribeirão Preto, SP, 1999.

19. Passos AD, Gomes UA, Figueiredo JF, Nascimento MM, Oliveira JM, Gaspar AM. Influência da migração na prevalência de marcadores sorológicos de hepatite B em comunidade rural. 1Análise da prevalência segundo local de nascimento. Revista de Saúde Pública 27:30-35, 1993.

20. Scharschmidt BF, Held MJ, Hollander HH, Read AE, Lavine JE, Veereman G, McGuire RF, Thaler MM. Hepatitis B in patients with HIV infection: relationship to AIDS and patient survival. Annals Internal of Medicine 117:837-838, 1992.

21. Schreeder MT, Thompson SE, Hadler SC, Berquist KR, Zaidi A, Maynard JE, Ostrow D, Judson FN, Braff EH, Nylund T, Moore Jr
JN, Gardner P, Doto IL, Reynolds G. Hepatitis B in homosexual men: prevalence of infection and factors related to transmission. Journal of Infectious Disease 146:7-15, 1982.

22. Sherlock S. Doenças do fígado e do sistema biliar. Guanabara Koogan, Rio de Janeiro, 1991.

23. Ter Meulen JT, Wittkowski KM, Kidenya JJ, Pöllath M, Dörries R, Fleischer K, Dietz K, Ter Meulen V. Evaluation of seroepidemiological associations between HIV-infection, hepatitis $B$ and other sexually transmitted diseases in african patients. European Journal Epidemiology 5:158-163, 1989.

24. Torres PRR, Mendes CGF. Marcadores virais no diagnóstico da hepatite. Byk Química, Rio de Janeiro, 1995 